\title{
Local sourcing in astronomy
}

\author{
In the age of huge telescopes involving many wealthy nations, we mustn't overlook regional telescopes that help \\ countries address their specific development needs.
}

$T^{\text {nis }}$ he announcement of yet another delay in the launch of the James Webb Space Telescope (JWST) a few weeks ago has the astronomical community reeling, with potential repercussions felt from the planning of the Wide Field Infrared Survey Telecope to the upcoming USA decadal survey and everything in between. JWST is one of several billion-dollar-class international projects currently underway - others include the European Extremely Large Telescope (E-ELT), the Giant Magellan Telescope and the Square Kilometre Array (SKA) - on which much of the future of astronomical research seems to rely. In an era of pooling resources under monolithic mega-projects, what is the role of the individual astronomical observatory? What can smaller communities contribute to the scientific discourse without access to the latest and biggest facilities?

In this issue, we turn our focus to four regional astronomical communities: Uzbekistan, Thailand, Morocco and Iran. Their gross domestic products and investments in fundamental science are eclipsed by those of established economies such as the USA and the European Union. Their budgets effectively bar them from active participation in the aforementioned megaprojects, but smaller telescopes are better able to address specific regional needs, and, more importantly, they are generally available, unlike the mega-projects that are few in number and highly competitive in terms of time allocation.

The Maidanak observatory in Uzbekistan (see the Comment by Shuhrat Ehgamberdiev) is an example of a small but active facility. Owing to its key location, the observatory has been involved in a number of scientific projects that link the European and the Asian continents, much like the Silk Road once did for trade and learning. Thailand on the other hand is an emerging market with small observatories cropping up across the country (see the Comment by Boonrucksar Soonthornthum). With plans for a new $40-\mathrm{m}$ radio telescope, a new generation of astronomers will soon be trained there. Morocco, building on a long tradition of astronomy in the Islamic world, is also making plans to expand its observing capabilities through a new telescope and associated instruments (see the Comment by Zouhair Benkhaldoun). The participation of the Oukaïmden Observatory in Morocco in the discovery and characterization of the
TRAPPIST-1 system is a prime example of high-impact science made by modest means. Last, but not least, Iran has been investing in a national observatory for both astronomical research and education (see the Mission Control by Habib Khosroshahi). In particular, training for postgraduates in observational astronomy and its technological applications may help keep Iranian graduates from leaving the country.

These smaller telescopes do not necessarily exist in isolation. As part of wider surveys, they improve the effective coverage and resolution. As training telescopes, they offer hands-on practical know-how for budding astronomers, preparing them for the intricacies and technical challenges of one day using the big league of the ELTs. As pathfinders for narrow-beam observatories such as the Thirty Meter Telescope and JWST, they provide precursor exploration of the sky for worthy targets. For example, the 8-m Subaru telescope, operated by the National Astronomical Observatory of Japan (NAOJ), has long been a prolific facility excelling in widefield area surveys. It will be critical to maintain such smaller telescopes in the age of the ELTs.

And while education and training is an important factor for sustainable development more globally, technology transfer and the creation of applications for regional solutions is equally critical. Going back to Iran as an extreme example, each year the country produces 750,000 university graduates, with more than $70 \%$ of science, technology, engineering and mathematics (STEM) graduates being women. They have no shortage of potential female scientists; however, the economy cannot employ everyone, hence, the well-documented 'brain drain'. Stemming this flow is high on the government agenda. Building a world-class optical telescope in Iran is therefore not mere nostalgia for the days when Persia was a world leader in astronomy, but a necessity to help absorb graduates and provide them with the skills and opportunities for collaboration with global researchers.

Moreover, astronomy can be viewed as a 'gateway' for people to get into science and technology. The International Astronomical Union certainly takes this approach with their Office of Astronomy for Development. Their mission is "to help further the use of astronomy, including its practitioners, skills and infrastructures, as a tool for development by mobilizing the human and financial resources necessary in order to realize the field's scientific, technological and cultural benefits to society." We need only look at the charge-coupled device (CCD) camera developed for astronomy to see the potential benefits to society. Large-scale astronomical surveys are also generating data at an unprecedented rate, leading to advances in bigdata handling and a generation of scientists working on machine-learning algorithms to make sense of the information overload. Applications in medicine, communications and climate modelling, for instance, can help other countries monitor various data and help inform effective government policies.

And finally, sustainable development and improvements to quality of life will not only help local regions but will improve things on a global scale. As in any other human venture, diversifying the roster of players is key in continued growth and meaningful discoveries. Uzbekistan, Thailand, Morocco and Iran are only a few examples of emerging markets with a largely untapped potential of ideas and of researchers that could rejuvenate scientific thinking and practices in the 'developed' world. Cross-pollination of ideas through truly international collaborations is the only way forward to ensure equal participation and universal access to not only the consumption of knowledge but also its production.

But a sustained political will to fund astronomy as an agent of development must be long term - robust against a change of government. In the case of Brazil, its full membership in the European Southern Observatory (ESO) was agreed by one government but never ratified by the next two, and it has recently been suspended from ESO. Meanwhile, China has created a domestic planetary science community in a matter of ten years. It is working with many international partners, particularly for its upcoming Jupiter mission (see the Comment by Yong Wei et al.), and a new observatory in Tibet will support space missions of ESA and NASA. Now, more than ever, we need to build and maintain an environment of mutual benefit, not just between wealthy nations but across a variety of countries, especially involving those emerging markets poised to supply the next generation of scientists and engineers.

Published online: 1 May 2018 https://doi.org/10.1038/s41550-018-0479-z 